\title{
Mitochondrial ferritin protects the murine myocardium from acute exhaustive exercise injury
}

\author{
Wenyue Wu ${ }^{1,3}$, Shiyang Chang ${ }^{1,3}$, Qiong $\mathrm{Wu}^{1,3}$, Zhifang $\mathrm{Xu}^{1,2}$, Peina Wang ${ }^{1}$, Yaru $\mathrm{Li}^{1}$, Peng Yu ${ }^{1}$, Guofen Gao ${ }^{1}$, Zhenhua Shi ${ }^{1}$, \\ Xianglin Duan ${ }^{1}$ and Yan-Zhong Chang,
}

\begin{abstract}
Mitochondrial ferritin (FtMt) is a mitochondrially localized protein possessing ferroxidase activity and the ability to store iron. FtMt overexpression in cultured cells protects against oxidative damage by sequestering redox-active, intracellular iron. Here, we found that acute exhaustive exercise significantly increases FtMt expression in the murine heart. FtMt gene disruption decreased the exhaustion exercise time and altered heart morphology with severe cardiac mitochondrial injury and fibril disorganization. The number of apoptotic cells as well as the levels of apoptosis-related proteins was increased in the $\mathrm{FtMt}^{-1-}$ mice, though the ATP levels did not change significantly. Concomitant to the above was a high 'uncommitted' iron level found in the $\mathrm{FtMt}^{-l-}$ group when exposed to acute exhaustion exercise. As a result of the increase in catalytic metal, reactive oxygen species were generated, leading to oxidative damage of cellular components. Taken together, our results show that the absence of FtMt, which is highly expressed in the heart, increases the sensitivity of mitochondria to cardiac injury via oxidative stress.
\end{abstract}

Cell Death and Disease (2016) 7, e2475; doi:10.1038/cddis.2016.372; published online 17 November 2016

Localized increases of reactive oxygen/nitrogen species (ROS/RNS) in the body is a primary cause for multiple health problems such as cardiovascular diseases, diabetes, cancer, and neurodegenerative diseases (Alzheimer's, Parkinson's, and Huntington). ${ }^{1-5}$ Indeed, relatively common cardiovascular diseases, such as atherosclerosis, hypertension, myocardial infarction, cardiac hypertrophy, and cardiomyopathy, are ascribed to increased oxidative stress. ${ }^{6-11}$

$\mathrm{FtMt}$, a recently discovered $\mathrm{H}$-ferritin-like protein, is present only in mitochondria. It was found that FtMt is strongly expressed in the testis, particularly in the spermatocytes, and also in the heart, kidney, Purkinje cells, ${ }^{12-14}$ and some other neurons, ${ }^{15,16}$ and generally in cells with high respiratory activity. Importantly, the FtMt transcript does not contain a functional iron responsive element (IRE) ${ }^{17}$ and thus, FtMt expression is not post-transcriptionally controlled by intracellular iron levels in the same way as that of $\mathrm{H}$-ferrtin. The tissue distribution pattern suggests that the major role of FtMt may be to protect mitochondria from iron-dependent oxidative damage. ${ }^{18,19}$ Our previous studies have shown that FtMt exerts a neuroprotective effect against 6-hydroxydopamine (6-OHDA)-induced dopaminergic cell damage ${ }^{18}$ and that FtMt is involved in the pathology of $A D$ where it may have a neuroprotective role by reducing oxidative stress. ${ }^{19}$ Recent research suggests FtMt may have a protective role in a number of mild pathophysiological conditions in the heart. ${ }^{20}$ Because FtMt is highly expressed in cardiac muscle, we hypothesize that the protein may protect mitochondria against oxidative damage from injury induced by acute exhaustive exercise (AEE), which is commonly used as an oxidative stress model. ${ }^{21}$

To examine the role of FtMt in the myocardium, we subjected age-matched (10- to 12-week-old) $\mathrm{FtMt}^{+/+}$and $\mathrm{FtMt}^{-1-}$ mice to AEE and evaluated the resultant myocardial injury. We found that $A E E$ induced an increase in intracellular 'uncommitted' iron, accompanied by oxidative stress and activation of the caspase cascade. These effects were exacerbated by FtMt deficiency, leading to increased myocardial injury and apoptosis.

\section{Results}

AEE induces FtMt expression in the murine myocardium. FtMt is critical for maintaining intracellular iron homeostasis in multiple cell types and organs. ${ }^{22}$ We performed immunoblotting to test whether FtMt is expressed in the mouse myocardium after AEE. As shown in Figures $1 a$ and b, we found that FtMt expression indeed significantly increased in the heart. We also measured FtMt mRNA levels in the mouse myocardium using the quantitative real-time PCR method. AEE treatment significantly increased FtMt mRNA expression in the mouse hearts (Figure 1c).

FtMt gene disruption affects myocardial structure and function. Time to exhaustion is a measure of athletic capacity. The time to exhaustion in $\mathrm{FtMt}^{-/-}$mice was decreased when compared with $\mathrm{FtMt}^{+/+}$mice (Figure 2a). To examine the structural differences between normal- and

\footnotetext{
${ }^{1}$ Laboratory of Molecular Iron Metabolism, The Key Laboratory of Animal Physiology, Biochemistry and Molecular Biology of Hebei Province, College of Life Science, Hebei Normal University, Shijiazhuang 050024, China and ${ }^{2}$ The 3rd Hospital of Hebei Medical University, Shijiazhuang 050051, China

${ }^{*}$ Corresponding author: Yan-Zhong Chang, Laboratory of Molecular Iron Metabolism, The Key Laboratory of Animal Physiology, Biochemistry and Molecular Biology of Hebei Province, College of Life Science, Hebei Normal University, 20, Nanerhuan Eastern Road, Shijiazhuang 050024, China. Tel: +86 31180786311 ;

Fax: +86 311 80786311; E-mail: frankyzchang@yahoo.com.hk

${ }^{3}$ These authors contributed equally to this work.

Received 28.7.16; revised 13.9.16; accepted 11.10.16; Edited by C Munoz-Pinedo
} 
a

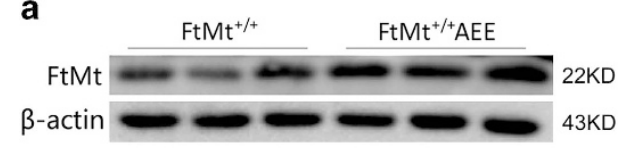

b

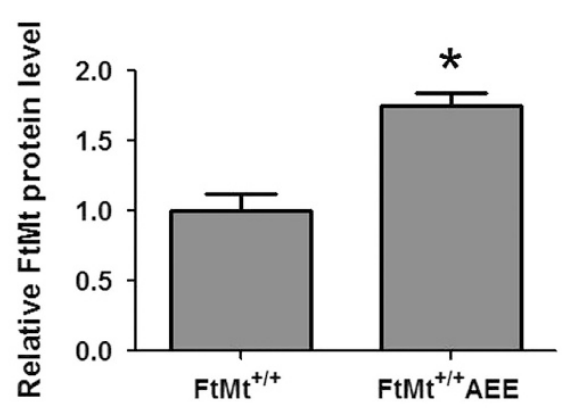

C

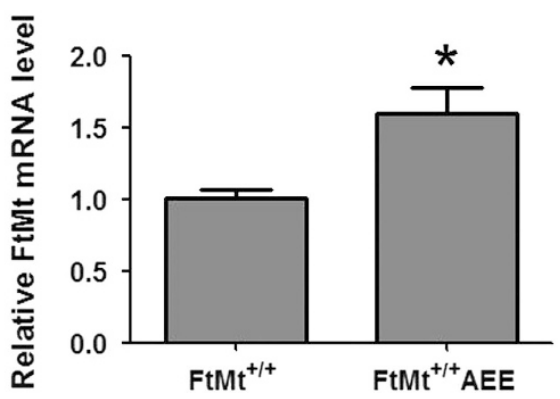

Figure 1 Increased FtMt expression in myocardia of wild-type mice after AEE. FtMt protein was detected by immunoblotting (a), normalized expression levels (b). Data were normalized by $\beta$-actin, FtMt mRNA expression (c) was detected by real-time PCR. The data are expressed as the mean \pm S.D. ${ }^{*} P<0.05$ versus $\mathrm{FtMt}^{+/+}$group. $n=6$
FtMt-deficient cardiomyocytes, we performed transmission electron microscopy in $\mathrm{FtMt}^{+/+}$and $\mathrm{FtMt}^{-/-}$cardiomyocytes of untreated mice or those subjected to AEE (Figure $2 b$ ). In the hearts of the latter, we observed significant mitochondrial vacuolation. Importantly, fibril organization was comparable between the $\mathrm{FtMt}^{+/+}$and $\mathrm{FtMt}^{+/+} \mathrm{AEE}$ groups. In contrast, the hearts of $\mathrm{FtMt}^{-/-}$mice exhibited mitochondria with slightly disrupted cristae, after experiencing AEE. In addition, the tissue structural damage was more severe with condensation and fragmentation of most myofibrils in some fields. Furthermore, mitochondrial damage was more evident in zones in which the cristae were absent. Functional changes (Figure 2c) accompanied the observed structural damage, when we assayed the ATP content of the cardiac tissue in the four groups, we observed a significant decrease in $\mathrm{FtMt}^{-/-}$ mice subjected to AEE.

Apoptosis is increased in cardiomyocytes of $\mathrm{FtMt}^{-1-}$ mice. We used the TUNEL method to detect apoptosis after exposing mice to AEE (Figure 3a). The number of apoptotic cells (Figure $3 \mathrm{~b}$ ) in the $\mathrm{FtMt}^{+/+} \mathrm{AEE}$ group was about four times greater than that in the $\mathrm{FtMt}^{+/+}$group, whereas the amount of apoptotic cells in the $\mathrm{FtMt}^{-/}-\mathrm{AEE}$ group was more than three-fold that in the $\mathrm{FtMt}^{+/+} \mathrm{AEE}$ group. There was also a marked increase in the $\mathrm{FtMt}^{-/} \mathrm{AEE}$ group compared with the $\mathrm{FtMt}^{-/-}$group. Together, these results suggest that the presence of FtMt protects against AEE-induced apoptosis.

Proteins of the Bcl-2 family include critical regulators of programmed cell death. Bcl-2 has the role of promoting cell survival. Increased expression of the pro-apoptotic protein, Bax, can promote cell death by activating elements of the caspase pathway, ${ }^{23}$ especially caspase- $-3{ }^{24}$ Thus, Bcl-2/Bax a

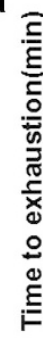

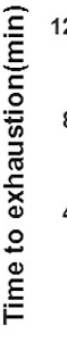
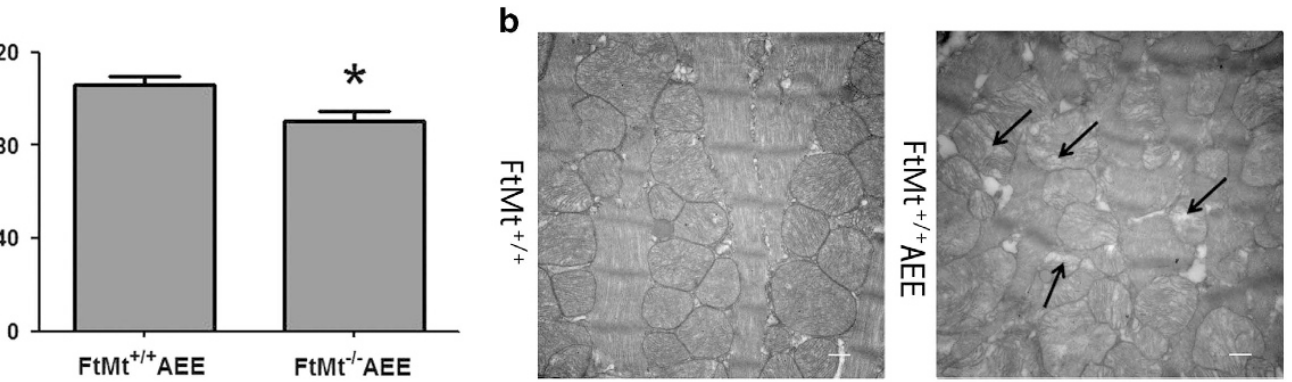

c
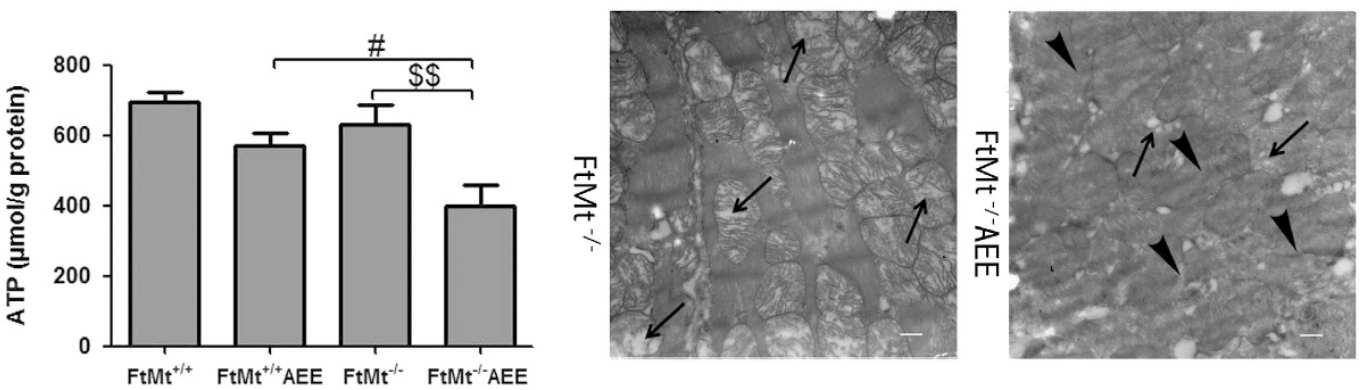

Figure 2 The role of FtMt in time of AEE and morphology, and function of mouse heart. (a) The statistics of exhaustive time in FtMt ${ }^{+/+} \mathrm{AEE}$ and FtMt ${ }^{-/-} \mathrm{AEE}$ mice. Values are presented as the mean \pm S.D. ${ }^{*} P<0.05$ versus $\mathrm{FtMt}^{+/+} \mathrm{AEE}$ group. $n=6$. (b) Transmission electron microphotographs exhibiting the heart morphology after AEE. Arrows indicate damage/absence of cristae; arrowheads indicate fibril disarrangement. The picture is magnified $20 \times 10^{3}$ times, scale bars $=500 \mathrm{~nm}$. Three animals for each group and three sections for each sample were examined, and representative images are shown. (c) ATP is downregulated in $\mathrm{FtMt}^{+/+}$and FtMt $^{-/-}$after AEE. Values are presented as the mean \pm S.D. ${ }^{\#} P<0.05$ versus FtMt ${ }^{+/+} A E E$ group, ${ }^{\$} P<0.01$ versus FtMt $^{-1-}$ group. $n=4$ 


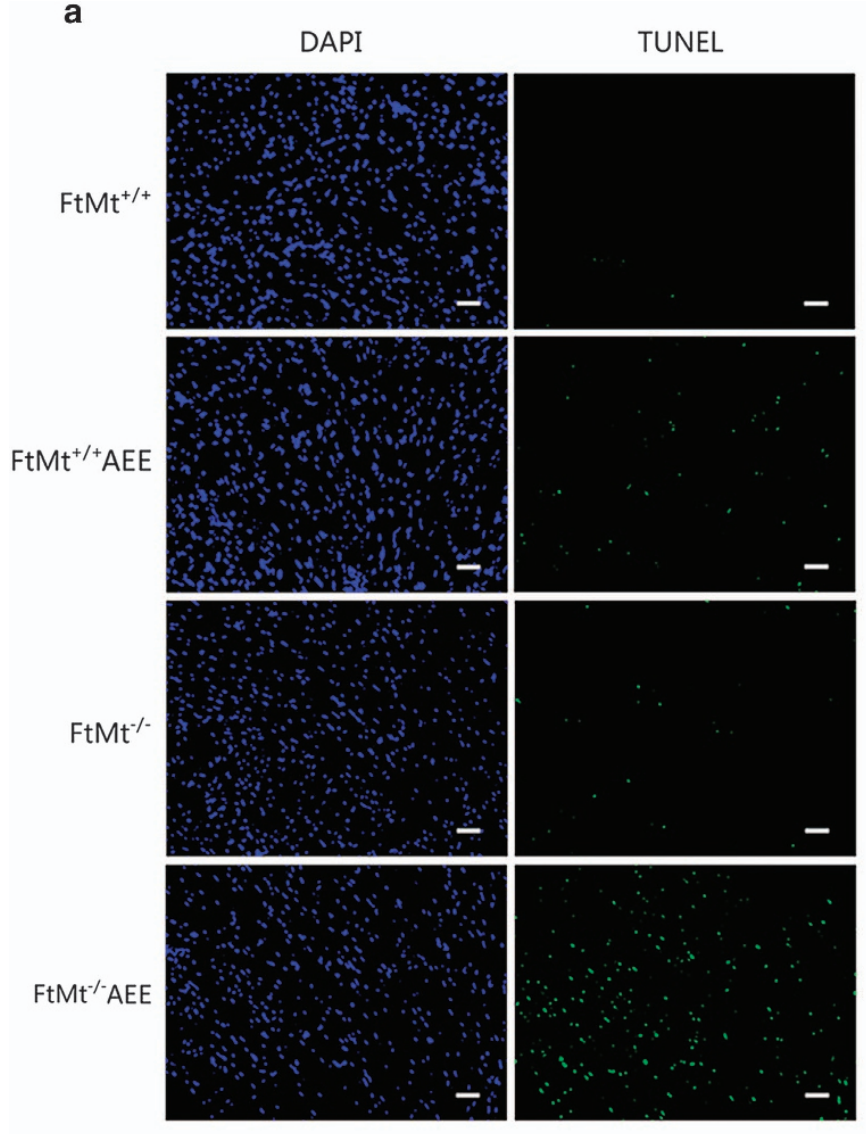

b

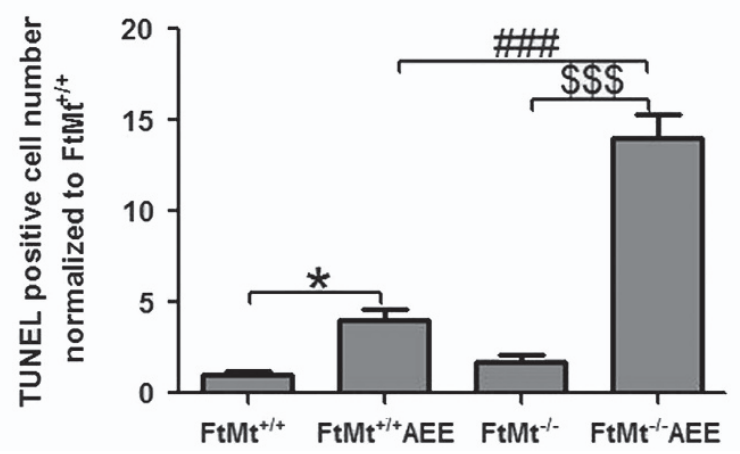

C

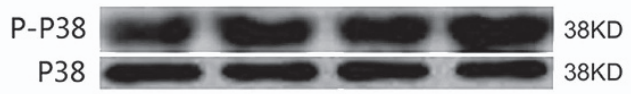

d

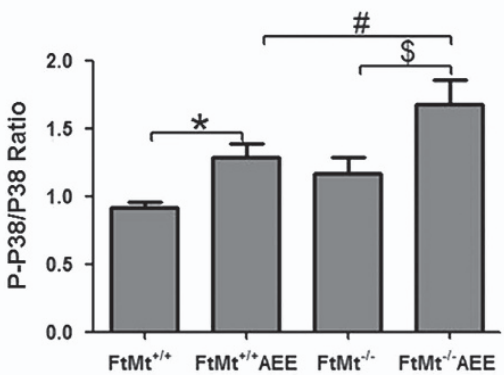

e

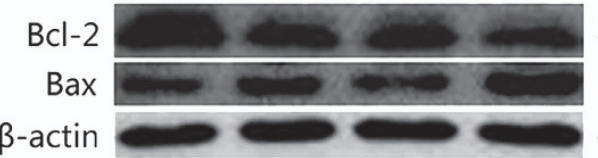

26KD

$23 K D$

$43 K D$

$\mathbf{f}$
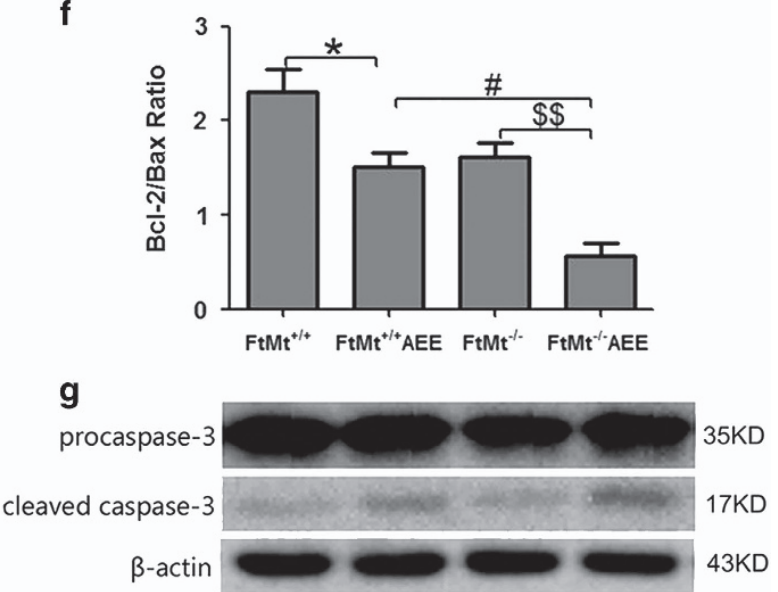

h

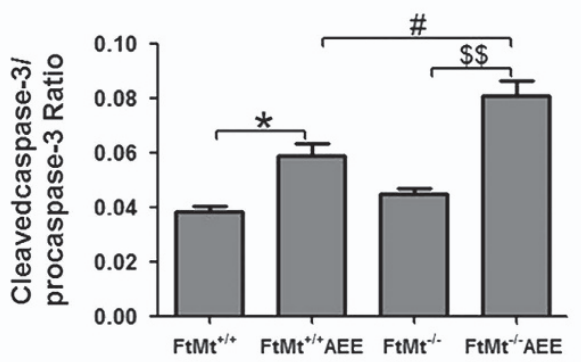

Figure 3 FtMt gene knockout aggravates AEE-induced myocardial apoptosis. (a) Apoptotic cells in the cardiac apex were assayed by TUNEL staining. The picture is magnified 200 times, scale bars $=50 \mu \mathrm{m}$. (b) The normalized amounts of apoptotic cells. Values are presented as the mean \pm S.D. ${ }^{*} P<0.05$ versus $\mathrm{FtMt}^{+/+}$group, ${ }^{\# \# \# ~} P<0.001$ versus $\mathrm{FtMMt}^{++} \mathrm{AEE}$ group, ${ }^{\$ \$} P<0.001$ versus FtMt ${ }^{-1-}$ group. $n=3$. Immunoblotting detected P38 and P-P38 (c), Bcl-2 and Bax (e), caspase-3, and cleaved caspase-3 (g) in the myocardium; the ratio of P-P38/P38 (d), Bcl-2/Bax (f), cleaved caspase-3/procaspase-3 (h) in the myocardium of FtMt ${ }^{+/+}, \mathrm{FtMt}^{-/-}, \mathrm{FtMt}^{+/+} \mathrm{AEE}$, and FtMt ${ }^{-/}-\mathrm{AEE} \mathrm{groups}^{-}$ were calculated. The data are expressed as the mean \pm S.D. ${ }^{*} P<0.05$ versus $\mathrm{FtMt}^{+/+}$group, ${ }^{\#} P<0.05$ versus $\mathrm{FtMt}^{+/+} \mathrm{AEE}$ group, ${ }^{\$} P<0.05,{ }^{\$ \$} P<0.01$ versus $\mathrm{FtMt}{ }^{-/-}$ group. $n=6$

levels are widely used as a proxy for the degree of apoptosis. ${ }^{18}$ The activation of MAPK is often seen in oxidative stressinduced cell death. ${ }^{25}$ MAPK (a.k.a., p38 MAP kinase or P38) is activated by phosphorylation; a high P-P38/P38 ratio can simultaneously promote Bax expression and decrease Bcl-2 levels. AEE significantly increased the $\mathrm{P}-\mathrm{P} 38 / \mathrm{P} 38$ ratio (Figures $3 \mathrm{c}$ and $\mathrm{d}$ ) with the expected, downstream consequences of decreased ratios of $\mathrm{Bcl}-2$ to $\mathrm{Bax}$ (Figures $3 e$ and $\mathrm{f}$ ) and elevated levels of cleaved caspase-3 (Figures $3 g$ and $h$ ). Out of all the tested groups, the largest changes in the 
a

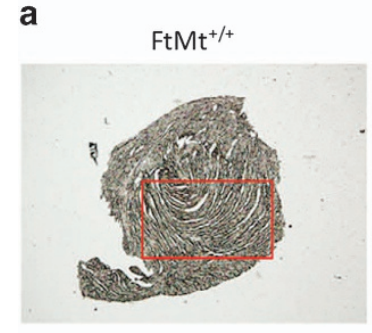

FtMt $^{-/-}$

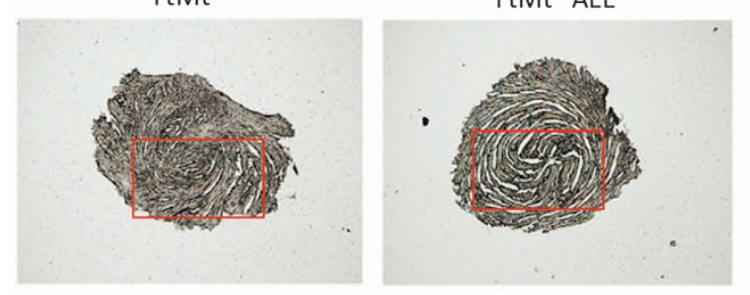

b
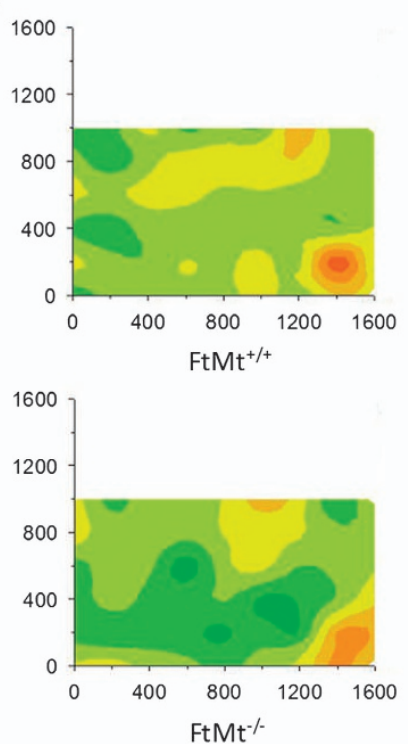
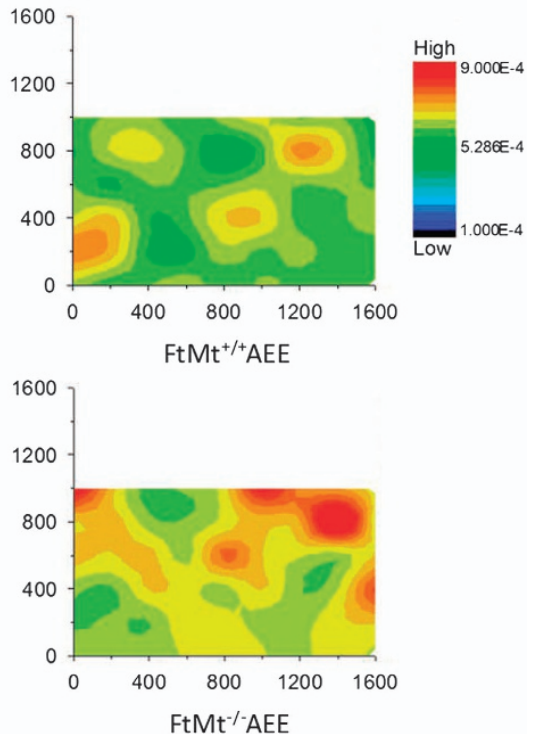

Figure 4 FtMt gene knockout increased mouse heart calcium levels after AEE. (a) Depiction of the scan measurement area in the slice of the cardiac apex region. (b) Calcium distribution in a representative position (area measurement (AM)-1.6 $\times 1.0 \mathrm{~mm}$ ) of the cardiac apex of FtMt ${ }^{+/+}, \mathrm{FtMt}^{-1-}, \mathrm{FtMt}^{+/+} \mathrm{AEE}$, and FtMt ${ }^{-/-} \mathrm{AEE}$ mice, detected by SR-XRF

$\mathrm{Bcl}-2 / \mathrm{Bax}$ ratios and cleaved caspase-3 levels were observed in the $\mathrm{FtMt}^{-/}$AEE mice.

Calcium levels increased in the $\mathrm{FtMt}^{-1-}$ mouse myocardium. Calcium activates various signal transduction pathways, including those that mediate apoptosis, so it is no surprise that dysregulated calcium metabolism can induce apoptosis. In fact, elevated unsequestered calcium levels can be an early indicator of apoptosis. ${ }^{26}$ We utilized synchrotron radiation $\mathrm{X}$-ray fluorescence (SR-XRF) to directly detect the calcium distribution in the myocardia of $\mathrm{FtMt}^{+/+}$and $\mathrm{FtMt}^{-/-}$ mice with or without exposure to acute oxidative stress. Our data show (Figure 4) that calcium levels increased in the myocardia of all groups after AEE, but was especially elevated in the $\mathrm{FtMt}^{-/-} \mathrm{AEE}$ group. These results demonstrate that aberrant calcium metabolism caused by AEE may be activating apoptosis the in the myocardia.

Mice deficient in FtMt possess elevated myocardial oxidative stress. To study whether increased levels of oxidative stress are responsible for the increased oxidative damage and apoptosis observed in the myocardium, we examined the antioxidant capacity in each group. Superoxide dismutase (SOD) is an antioxidant enzyme that can mitigate the accumulation of ROS, whereas malondialdehyde (MDA) is a consequence of excess ROS and whose levels are commonly viewed as an indicator of lipid peroxidation. Compared with the $\mathrm{FtMt}^{+/+}$group, the SOD activity (Figure 5a) was lower while the levels of MDA (Figure 5b) were increased in the other three groups. In addition, the lowest SOD activity and the highest level of MDA both appeared in the myocardium of the $\mathrm{FtMt}^{-/-} \mathrm{AEE}$ group. The level of MDA in the $\mathrm{FtMt}^{-/}$AEE group was about four-fold the level of that in the $\mathrm{FtMt}^{+/+}$group, whereas the amount of MDA in the $\mathrm{FtMt}^{-/-} \mathrm{AEE}$ group was about three-fold greater than that of the $\mathrm{FtMt}^{+/+} \mathrm{AEE}$ group. We also found that the decreases in catalase, $\mathrm{NAD}(\mathrm{P}) \mathrm{H}$ :quinone oxidoreductase 1, and heme oxygenase 1 mRNA were greater between the $\mathrm{FtMt}^{-/-} \mathrm{AEE}$ and $\mathrm{FtMt}^{-/-}$groups, compared with those between the $\mathrm{FtMt}^{+/+} \mathrm{AEE}$ and $\mathrm{FtMt}^{+/+}$groups (Supplementary Figure 2). These findings are congruent with dramatically increased oxidative stress in the myocardium of $\mathrm{FtMt}^{-1-}$ mice subjected to AEE.

FtMt gene disruption results in altered myocardial iron metabolism after AEE. Transferrin receptor 1 (TfR1) and divalent metal transport 1 (DMT1, of which there are two variants, +IRE and -IRE) are major players in iron uptake, whereas ferroportin 1 (FPN1) is the only known cellular iron exporter. Ferritin, comprising $\mathrm{H}$-ferritin and $\mathrm{L}$-ferritin subunits, can sequester and store iron in a safe yet accessible form. ${ }^{27}$ By immunoblot analysis, we observed that AEE decreases the expression of both ferritin subunits ( $\mathrm{H}$ - and L-ferritin) (Figures $6 e$ and $f$ ) in the myocardium, but increases TfR1 expression (Figures $6 a$ and b). However, there is no difference in the expression of Fpn1, DMT1+IRE, or DMT1-IRE in any group (Figures 6a-d).

We next utilized SR-XRF to directly detect the iron distribution and content in the myocardium of the four groups. Our data (Figures $7 a$ and b) show that iron indeed accumulated in both the $\mathrm{FtMt}^{+/+} \mathrm{AEE}$ and $\mathrm{FtMt}^{-/-} \mathrm{AEE}$ groups. As elevated, 'uncommitted' iron is able to directly generate ROS through Fenton chemistry, our results show that aberrant iron metabolism may be involved in the oxidative stress brought about by AEE.

\section{Discussion}

Mitochondrially generated ROS are involved in a myriad of signaling and damaging pathways in different tissues. ${ }^{28}$ In 
a

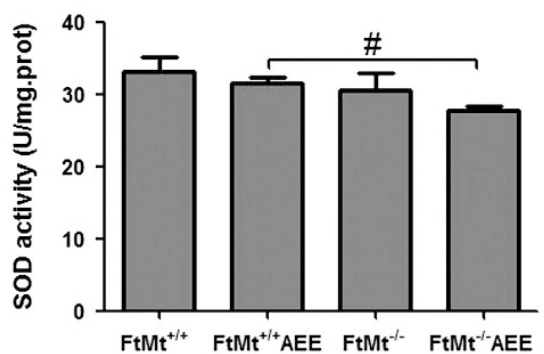

b

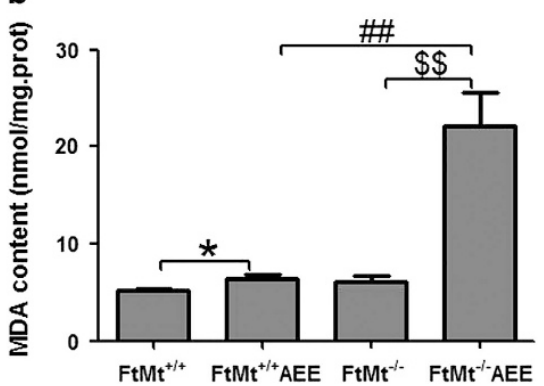

Figure 5 FtMt gene knockout increases oxidative stress. SOD activity (a) and the level of MDA (b) were detected in the myocardium of $\mathrm{FtMt}^{+/+}, \mathrm{FtMt}^{-/-}$, $\mathrm{FtMt}^{+/+} \mathrm{AEE}$, and $\mathrm{FtMt}^{-l-}$ AEE mice. Values are presented as the mean \pm S.D. ${ }^{*} P<0.05$ versus $\mathrm{FtMt}^{+/+}$group, ${ }^{\#} P<0.05,{ }^{\# \#} P<0.01$ versus $\mathrm{FtMt}^{+/+} \mathrm{AEE}$ group, ${ }^{\$ \$} \mathrm{P}<0.01$ versus $\mathrm{FtMt}^{-/-}$group. $n=5$

addition, mitochondria are an important target of ROS and RNS. ${ }^{29}$ FtMt is expected to protect the mitochondria from the accumulation of ROS; a hypothesis that has been confirmed by in vitro studies of cells that overexpress FtMt. ${ }^{19,30}$ Our previous experiments ${ }^{18,19}$ confirm FtMt has a protective role in cells by reducing oxidative damage, whereas recent research from others suggests FtMt may have a protective role under a number of not particularly severe pathophysiological conditions in the heart. ${ }^{22}$ However, the mechanism of the protective role of FtMt in the heart had not been revealed.

We used an AEE-induced oxidative stress model of cardiac injury in the present study. ${ }^{21}$ Our results show that AEE elevates FtMt protein levels in the mouse myocardium, providing a clue that FtMt may have an important role in maintaining myocardial function.

Previous studies have indicated AEE can give rise to oxidative stress. ${ }^{21,31}$ To evaluate the possible protective activity of FtMt in this context, we measured the time to exhaustion of $\mathrm{FtMt}^{+/+}$and $\mathrm{FtMt}^{-/-}$mice and found that the wild-type mice could exercise longer. Histological examination by electron microscopy revealed morphological abnormalities, including mitochondrial vacuolation, in hearts after mice of both genotypes were subjected to AEE. Strikingly, AEEaggravated $\mathrm{FtMt}^{-/-}$hearts showed considerable damage to mitochondrial cristae as well as myofibril disarrangement in some areas of the heart. Biochemical assays of heart functionality revealed decreased ATP content, indicating mitochondrial dysfunction. Together, these results show that FtMt has an important role in maintaining cardiac structure and function.

Apoptosis can be induced by oxidative stress in a caspasemediated pathway. ${ }^{32}$ Our data show that apoptosis was induced by AEE in both $\mathrm{FtMt}^{+/+}$and $\mathrm{FtMt}^{-/-}$mice, whereas $\mathrm{FtMt}^{-1-}$ mice exhibited a slightly increased amount of apoptosis without the exercise. Importantly, the $\mathrm{FtMt}^{-/-}$mice had a relatively high level of myocardial apoptosis after AEE. We also observed a reduction in the $\mathrm{Bcl}-2 / \mathrm{Bax}$ protein ratio, an enhanced P-P38/P38 ratio and an increase in caspase-3 protein in the myocardium after AEE. All three of these effects were exacerbated in the FtMt knockout mice. Taken together, our results strongly suggest that a bona fide apoptotic signal transduction pathway is initiated following AEE.
How does acute oxidative stress induce apoptosis and damage in the heart? Our results suggest AEE leads to the generation of ROS and subsequent oxidative damage through increased lipid peroxidation and the release of intracellular $\mathrm{Ca}^{2+}$, which is a pro-apoptotic factor. In addition, previous studies have shown that FtMt knockdown induces oxidative damage and stimulates apoptosis in the hippocampus after treatment with $\mathrm{A} \beta \mathrm{.}^{19}$

Why did the FtMt gene knockout aggravate the oxidative injury? Metals, such as iron, copper, and zinc, have been shown to be key factors in biochemical reactions that produce free radicals, which lead to the peroxidation of cellular lipids and cellular injury or apoptosis. ${ }^{33}$ Our results show that, the total iron of myocardia was increased after AEE, FtMt gene knockout aggravated iron overloading, which may be the reason of oxidative injury. Serum iron was also found to increase in the $\mathrm{FtMt}^{-/}$AEE group (Supplementary Figure 3), suggesting that AEE also has an effect on cells that release iron, such as enterocytes and macrophages of the reticuloendothelial system.

In summary, our study shows that acute exhaustive stress gives rise to the formation of ROS that initiate lipid peroxidation, protein oxidation, and cytoplasmic $\mathrm{Ca}^{2+}$ accumulation, eventually leading to myocardial apoptosis and damage. Furthermore, the ROS generated under this stress modality may be potentiated by the accumulation of iron that occurs when cellular iron metabolism is thrown off balance. Our data indicate that normal mice can mitigate oxidative damage by upregulating FtMt protein levels, whereas the absence of FtMt leads to severe heart mitochondria and myofibril damage. The cardiomyocyte mitochondria in $\mathrm{FtMt}^{-/-}$mice are more sensitive to oxidative stress-mediated injury, ${ }^{20}$ which likely underlies the decrease in time to exhaustion, possibly due to myocardial damage. In addition, increased IL-6 indicates that an inflammatory reaction may result from FtMt gene inactivation, further aggravating the heart injury by AEE (Supplementary Figure 4). All our data suggest FtMt has a protective role against myocardial injury induced by AEE. Moreover, the results of our study represent the first in-depth evidence for a function of FtMt in vivo and points to FtMt as a potential therapeutic target to protect the heart from oxidative stress-induced cardiomyopathy. 
a

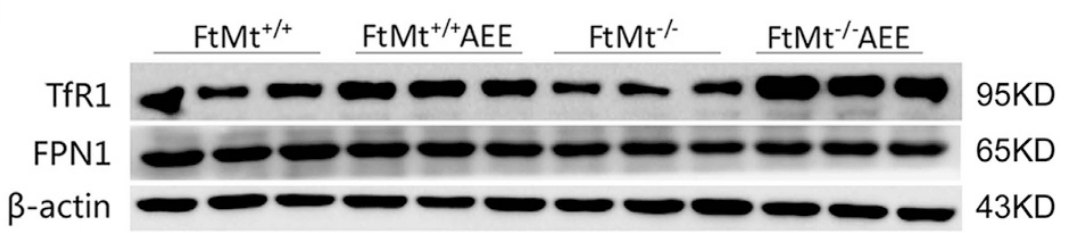

b
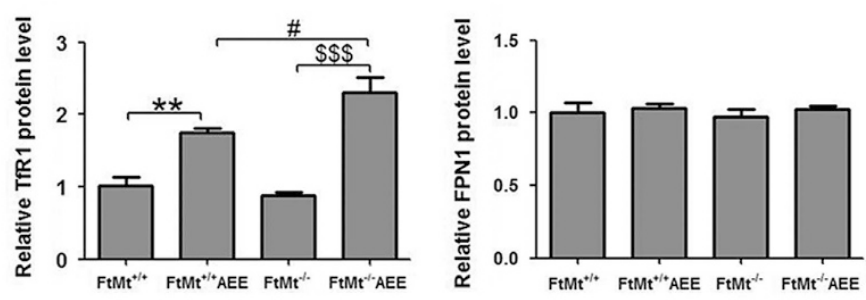

C
$\mathrm{FtMt}^{+/+}$
$\mathrm{FtMt}^{+/+} \mathrm{AEE}$
$\mathrm{FtMt}^{-/}$
$\mathrm{FtMt}^{-/-A E E}$

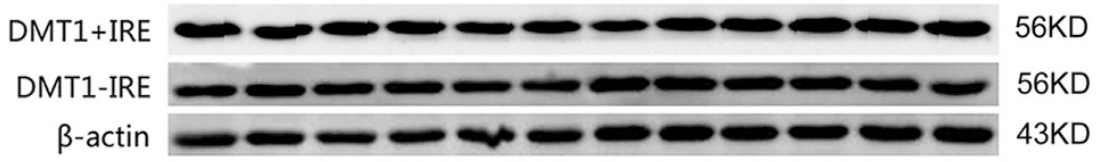

d
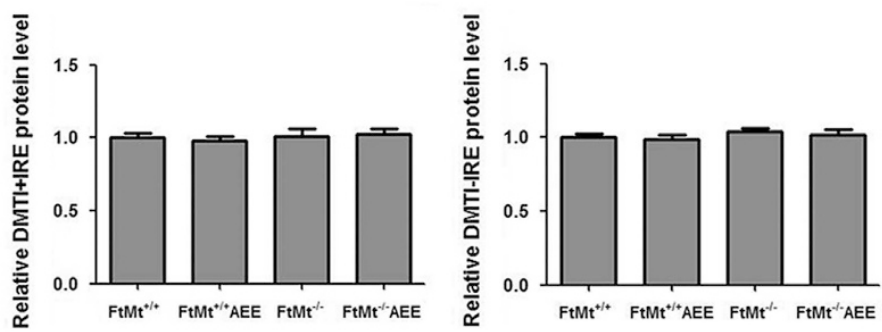

e

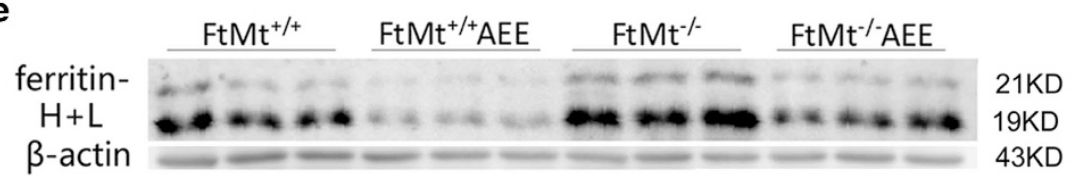

f

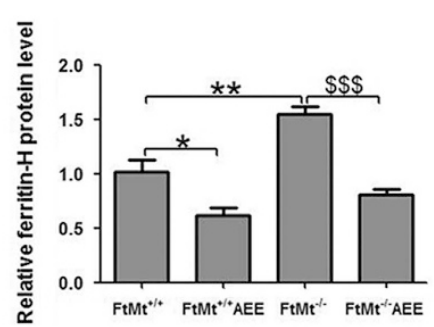

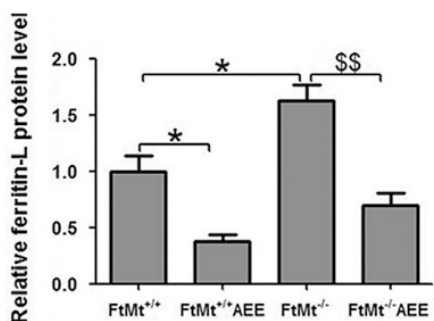

Figure 6 Immunoblotting detection of iron metabolism-related proteins in mice myocardia. (a, c, and e) Normalized levels of TfR1, FPN1, DMT1+IRE, DMT1-IRE, ferritin-H, ferritin-L, and $\beta$-actin in the myocardium of $\mathrm{FtMt}^{+/+}, \mathrm{FtMt}^{-/-}, \mathrm{FtMt}^{+/+} \mathrm{AEE}$, and $\mathrm{FtMt}^{-/}-\mathrm{AEE}$ group mice were detected by immunoblot. (b, d, and f) The TfR1, FPN1, DMT1+IRE, DMT1-IRE, ferritin- $\mathrm{H}$, and ferritin-L expression levels were normalized to $\beta$-actin and expressed as the mean \pm S.D. ${ }^{*} P<0.05,{ }^{* *} P<0.01$ versus FtMt ${ }^{+/+}$group, ${ }^{\#} P<0.05$ versus $\mathrm{FtMt}^{+/+} \mathrm{AEE}$ group, ${ }^{\$ \$} P<0.01,{ }^{\$ \$} P<0.001$ versus $\mathrm{FtMt}^{-1-}$ group. $n=6$

\begin{abstract}
Materials and Methods
Animals. Originally, the C57BL/6 wild and FtMt-null mice were generated by $\mathrm{Dr}$ M Fleming's group. ${ }^{34}$ The mice were bred and genotyped by PCR amplification of genomic DNA and total RNA (Supplementary Figure 1b) using the primers listed in Supplementary Table 1. Immunoblotting analysis for FtMt protein expression was further performed to confirm mouse genotypes (Supplementary Figures $1 \mathrm{c}$ and 1d). Mice were housed under conditions controlled for temperature $\left(22^{\circ} \mathrm{C}\right)$ and humidity (40\%), using a $12 \mathrm{~h}$ light (0700 to 1900), $12 \mathrm{~h}$ dark (1900 to 0700) cycle. ${ }^{35}$ Mice
\end{abstract}

were fed a standard rodent diet and water ad libitum. Age-matched $\mathrm{FtMt}^{+/+}$and $\mathrm{FtMt}^{-1-}$ male mice were subjected to AEE as described below. All procedures were carried out in accordance with the National Institutes of Health Guide for the Care and Use of Laboratory Animals, and were approved by the Animal Care and Use Committee of the Hebei Science and Technical Bureau in the PRC.

Antibodies and reagents. The following antibodies and reagents were used: FtMt (generous gift from Sonia Levi, Italy), $\beta$-actin (Alpha Diagnostic International, 


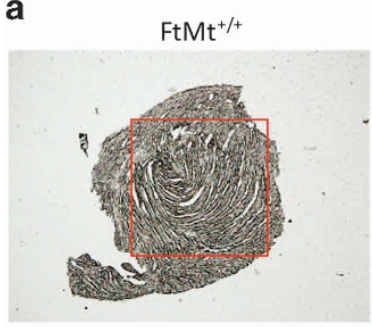

$\mathrm{FtMt}^{-1-}$

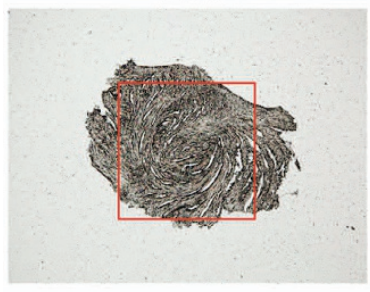

$\mathrm{FtMt}^{+/+} \mathrm{AEE}$

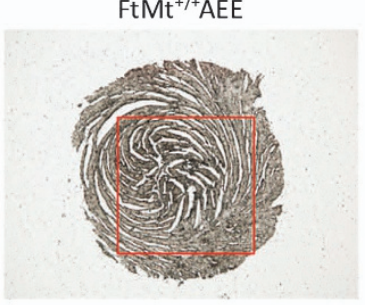

$\mathrm{FtMt}^{-1-\mathrm{AEE}}$

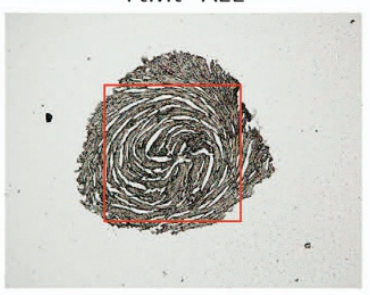

b
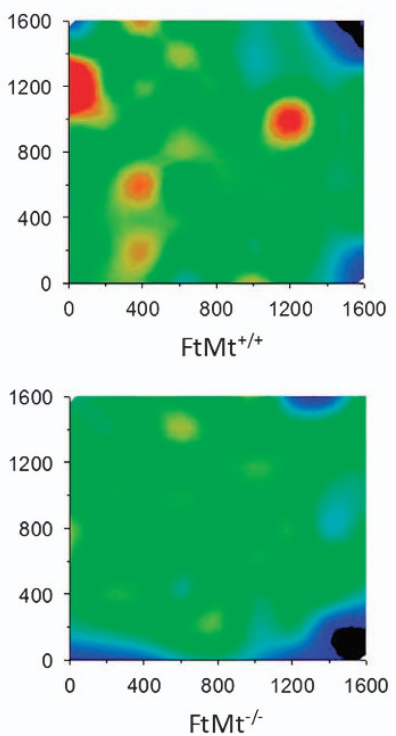

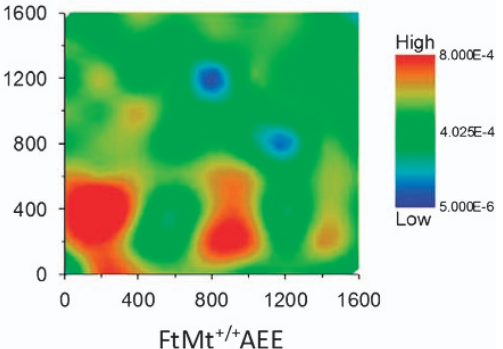

$\mathrm{FtMt}^{+/+} \mathrm{AEE}$

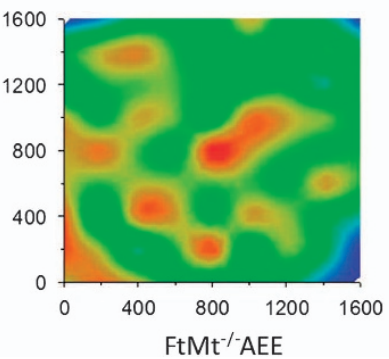

Figure 7 Iron distribution in mouse cardiac muscle. (a) Depiction of the scan measurement area in the slice of the cardiac apex region. (b) The iron distribution in a representative position (AM-1.6 $\times 1.6 \mathrm{~mm}$ ) of the cardiac apex of $\mathrm{FtMt}^{+/+}$, $\mathrm{FtMt}^{-/-}$, $\mathrm{FtMt}^{+/+} \mathrm{AEE}$, and FtMt ${ }^{-/-} \mathrm{AEE}$ mice as detected by SR-XRF

San Antonio, TX, USA), TfR1, FPN1 (Sigma-Aldrich, St Louis, MO, USA), DMT1+IRE and DMT1-IRE (Alpha Diagnostic International), Ferritin-H+L (Abcam Inc., San Francisco, CA, USA), P-P38 and P38 (Cell Signaling Technology, Danvers, MA, USA), Bcl-2 and Bax (Santa Cruz Biotechnology, Santa Cruz, CA, USA), caspase-3 (Cell Signaling Technology). Reagents for RNA extraction and reverse transcription were purchased from Takara (Dalian, China). Real-time RT-PCR quantification reagents were purchased from Genstar (Beijing, China). Protein expression was assessed by immunoblotting as previously described. ${ }^{13}$

Acute exhaustive exercise. $\mathrm{FtMt}^{+/+}$and $\mathrm{FtMt}^{-/-}$mice at 3 months of age ( $n=6-8 /$ group/experiment) were subjected to exercise on a gradient treadmill for 3 consecutive days at a $10^{\circ}$ incline $(15 \mathrm{~min}$ at $10 \mathrm{~m} / \mathrm{min}$ the first day; $30 \mathrm{~min}$ at $14 \mathrm{~m} / \mathrm{min}$ the second day; $45 \mathrm{~min}$ at $17 \mathrm{~m} / \mathrm{min}$ the third day). On the fourth day, the exercised group ran on the treadmill at an initial speed of $12 \mathrm{~m} / \mathrm{min}$ and incline $10^{\circ}$ for $7 \mathrm{~min}$, then at a speed of $14 \mathrm{~m} / \mathrm{min}$ for $5 \mathrm{~min}$. The speed was incremented by $2 \mathrm{~m} / \mathrm{min}$ every $2 \mathrm{~min}$ until the mice reached $22 \mathrm{~m} / \mathrm{min}$, and then the speed was incremented by $1 \mathrm{~m} / \mathrm{min}$ every $3 \mathrm{~min}$ until the mice reached their maximum velocity. ${ }^{21}$ Empirically, we observed that mice, like humans, are not all able to run at the same maximum velocities. Thus we defined the maximum velocity as the highest velocity at which the mouse would continue to run, without falling off the treadmill and being unable to hop back on, despite continued efforts and lack of clear signs of exhaustion. The mice were then run to exhaustion (i.e., run at maximum velocity until they would not remain on the treadmill despite gentle electric shocks). ${ }^{36,37}$ Immediately after exhaustion exercise, mice were killed and the left ventricule of hearts excised, processed/frozen in liquid nitrogen and stored at $-80^{\circ} \mathrm{C}$ for subsequent analysis.

Transmission electron microscopy. The tips of hearts were fixed in $3 \%$ glutaraldehyde in phosphate buffer $(0.1 \mathrm{M} ; \mathrm{pH} 7.4)$ overnight at $4{ }^{\circ} \mathrm{C}$, postfixed with $1 \%$ osmic acid in $0.1 \mathrm{M}$ phosphate buffer for $30 \mathrm{~min}$, dehydrated, and embedded in Araldite. Semi-thin sections, 2- $\mu \mathrm{m}$ thick, were stained with toluidine blue. For electron microscopy, ultrathin sections $(200 \mathrm{~nm})$ were obtained with an ultramicrotome (705902, Leica, Wetzlar, Germany), stained with uranyl acetate and lead citrate, and examined with a transmission electron microscope $(\mathrm{H}-7650$, Hitachi Ltd., Tokyo, Japan).

Determination of ATP content. The ATP content of heart tissue, from samples stored at $-80^{\circ} \mathrm{C}$, was measured by a colorimetric assay using a commercial kit (ATP colorimetric assay kit, Nanjing Jiancheng Bioengineering Institute, Nanjing, China) following the manufacturer's instructions.
Detection of apoptosis. The presence of apoptosis in the myocardium was assessed by the TUNEL method, ${ }^{38}$ as previously described. ${ }^{14}$ Nuclei were stained with DAPI. The number of TUNEL-DAPI-positive cardiomyocytes was counted using the same quantification method as described previously for counting. ${ }^{39}$ The counting area was located in the same position of the myocardium in all groups. For each group, quantification was performed in sections from three different mice, and the data are provided as the number of TUNEL-positive cells compared with that of the control group.

Quantitative real-time PCR. The relative purity of isolated total RNA was assessed spectrophotometrically and the ratio of A260/A280 nm exceeded 1.9 for all preparations. Total RNA $(1 \mu \mathrm{g})$ was reverse transcribed in a $20 \mu \mathrm{l}$ reaction and $1 \mu \mathrm{l}$ cDNA were then used as the template for real-time PCR with SYBR Green (GenStar). PCR amplification was performed with the BIO-RAD CFX Connect RealTime System (Hercules, CA, USA) with the following cycling parameters: $95^{\circ} \mathrm{C}$ for $10 \mathrm{~s}$, followed by 40 cycles of $95^{\circ} \mathrm{C}$ for $15 \mathrm{~s}$ and then $60^{\circ} \mathrm{C}$ for $1 \mathrm{~min}$. Expression of the target gene was determined by normalizing to the respective $\beta$-actin levels. Each amplification was repeated three times and the data were averaged. The forward primer of the FtMt sequence is: $5^{\prime}$-ATTTCCTTCGCCAGTCCC-3' and the reverse primer of FtMt is $5^{\prime}$-CCGCATTCCCAGTCATCT-3'; the $\beta$-actin forward primer sequence is $5^{\prime}$-AGGCCCAGAGCAAGAGAGGTA-3' and reverse primer sequence is $5^{\prime}$-TCTCCATGTCGTCCCAGTTG-3'.

Measurements of oxidative stress. MDA, a reliable marker of lipid peroxidation, was measured using the MDA kit, according to the manufacturer's instructions (Nanjing Jiancheng Bioengineering Institute, China). SODs, enzymes which catalyze the dismutation of superoxide into oxygen and hydrogen peroxide to provide important antioxidant defense in cells performing oxidative metabolism, were measured by a SOD kit according to the manufacturer's instructions (Nanjing Jiancheng Bioengineering Institute, China). The levels of MDA and the SOD activity were determined in each group. The left ventricular region of the heart was homogenized in ice-cold saline. The homogenate was centrifuged at $3000 \times g$ at $4^{\circ} \mathrm{C}$ for $15 \mathrm{~min}$, and then the supernatant was used to determine SOD activity and MDA levels with a UV-vis spectrophotometer at wavelengths of 550 and $532 \mathrm{~nm}$, respectively.

Synchrotron radiation X-ray fluorescence. Under anesthesia with nembutal, animals were perfused with saline followed by $4 \%$ paraformaldehyde in $0.1 \mathrm{M}$ phosphate buffer. The myocardium was removed, postfixed for $1.5-4 \mathrm{~h}$ and then stored overnight in $30 \%$ sucrose. Serial coronal sections were cut at $30 \mu \mathrm{m}$ intervals on a freezing microtome. Equivalent slices from the same coordinates 
were fixed onto $3 \mathrm{~mm}$-thick Mylar films (polycarbonate) from each of the four groups of mice. The myocardial samples were clamped between two layers of cellophane, dried at room temperature, and stored in a vacuum desiccator before analysis by SR-XRF. The microdistributions of iron and calcium in mouse myocardial samples were collected in fluorescence mode at room temperature at the Beijing Synchrotron Radiation Facility (BSRF). The continuous synchrotron X-rays were monochromatized by a $\mathrm{Si}(111)$ double crystal. The sample was placed at a $45^{\circ}$ angle to the incident $\mathrm{X}$-ray beam, and $\mathrm{X}$-ray fluorescence was detected with a $50 \mathrm{~mm}^{2}$ silicon drift detector (Vortex, Hitachi High-Technologies Science America Inc, Northridge, CA, USA) oriented at a $90^{\circ}$ angle to the incident beam. A light microscope was coupled to a computer for sample viewing. A monochromatic SR with a photon energy of $10 \mathrm{keV}$ was used to excite the samples. The beam was adjusted with grating to about $38 \times 28 \mu \mathrm{m}^{2}$. The sample platform was moved along the $X$ and $Z$ directions at intervals of 38 and $28 \mu \mathrm{m}$, respectively. The XRF signals were collected for up to $2 \mathrm{~s}$ at each point. In order to correct the effect of the SR beam flux variation on the signal intensity, the peak areas of $\mathrm{Fe}$ and calcium were normalized to the current intensity $(10)$ in an ionization chamber, which was placed in front of the samples. The peak areas were used for estimating the relative iron and calcium content in the samples. ${ }^{35}$ The results were analyzed using Origin 8.0 (Northampton, MA, USA), and iron signals in the tip of the heart were compared among the different groups.

Data analysis. All the data are expressed as the mean \pm S.D. The statistical analysis of four groups difference was assessed by a one-way analysis of variance followed by a post hoc test. The student's $t$-test was performed to compare between the two groups data. Differences were considered significant for $P<0.05$. All the tests were performed with SPSS21.0 (Armonk, NY, USA).

\section{Conflict of Interest}

The authors declare no conflict of interest.

Acknowledgements. This work was supported by the National Science Foundation of China (31520103908, 31300898, and 31271473). We are grateful to Professor Sonia Levi for the gift of anti-FtMt antibody. We acknowledge the Beijing Synchrotron Radiation Facility for the beam time.

1. Collins AR, Lyon CJ, Xia X, Liu JZ, Tangirala RK, Yin F et al. Age-accelerated atherosclerosis correlates with failure to upregulate antioxidant genes. Circ Res 2009; 104: e42-e54

2. Du $Y$, Wooten MC, Gearing M, Wooten MW. Age-associated oxidative damage to the p62 promoter: implications for Alzheimer disease. Free Radic Biol Med 2009; 46: 492-501.

3. Jacob MH, Janner Dda R, Araújo AS, Jahn MP, Kucharski LC, Moraes TB et al. Redox imbalance influence in the myocardial Akt activation in aged rats treated with DHEA. Exp Gerontol 2010; 45: 957-963

4. Li M, Fukagawa NK. Age-related changes in redox signaling and VSMC function. Antioxid Redox Signal 2010; 12: 641-655.

5. Morrison CD, Pistell PJ, Ingram DK, Johnson WD, Liu Y, Fernandez-Kim SO et al. High fat diet increases hippocampal oxidative stress and cognitive impairment in aged mice: implications for decreased Nrf2 signaling. J Neurochem 2010; 114: 1581-1589.

6. Capell BC, Collins FS, Nabel EG. Mechanisms of cardiovascular disease in accelerated aging syndromes. Circ Res 2007; 101: 13-26.

7. Hazzard WR, Ettinger WH Jr. Aging and atherosclerosis: changing considerations in cardiovascular disease prevention as the barrier to immortality is approached in old age. Am J Geriatr Cardiol 1995; 4: 16-36.

8. Rhoades DA, Welty TK, Wang W, Yeh F, Devereux RB, Fabsitz RR et al. Aging and the prevalence of cardiovascular disease risk factors in older American Indians: the Strong Heart Study. J Am Geriatr Soc 2007; 55: 87-94.

9. Segal BL, Tecce MA, Sherman FT. Cardiovascular disease and the aging U.S. population. Geriatrics 2003; 58: 43.

10. Waller BF, Bloch T, Barker BG, Roe SJ, Hawley DA, Pless JC et al. The old-age heart: aging changes of the normal elderly heart and cardiovascular disease in 12 necropsy patients aged 90 to 101 years. Cardiol Clin 1984; 2: 753-779.

11. Wissler RW, Robert L. Aging and cardiovascular disease: a summary of the Eighth Munster International Arteriosclerosis Symposium. Circulation 1996; 93: 1608-1612.
12. Corsi B, Cozzi A, Arosio P, Drysdale J, Santambrogio P, Campanella A et al. Human mitochondrial ferritin expressed in HeLa cells incorporates iron and affects cellular iron metabolism. J Biol Chem 2002; 277: 22430-22437.

13. Levi S, Corsi B, Bosisio M, Invernizzi R, Volz A, Sanford D et al. A human mitochondrial ferritin encoded by an intronless gene. J Biol Chem 2001; 276: 24437-24440.

14. Nie G, Sheftel $A D$, Kim SF, Ponka P. Overexpression of mitochondrial ferritin causes cytosolic iron depletion and changes cellular iron homeostasis. Blood 2005; 105: 2161-2167.

15. Drysdale J, Arosio P, Invernizzi R, Cazzola M, Volz A, Corsi B et al. Mitochondrial ferritin: a new player in iron metabolism. Blood Cells Mol Dis 2002; 29: 376-383.

16. Santambrogio P, Biasiotto G, Sanvito F, Olivieri S, Arosio P, Levi S. Mitochondrial ferritin expression in adult mouse tissues. J Histochem Cytochem 2007; 55: 1129-1137.

17. Shi ZH, Shi FF, Wang YQ, Sheftel AD, Nie G, Zhao YS et al. Mitochondrial ferritin, a new target for inhibiting neuronal tumor cell proliferation. Cell Mol Life Sci 2015; 72: 983-997.

18. Shi ZH, Nie G, Duan XL, Rouault T, Wu WS, Ning B et al. Neuroprotective mechanism of mitochondrial ferritin on 6-hydroxydopamine-induced dopaminergic cell damage: implication for neuroprotection in Parkinson's disease. Antioxid Redox Signal 2010; 13: 783-796.

19. Wu WS, Zhao YS, Shi ZH, Chang SY, Nie GJ, Duan XL et al. Mitochondrial ferritin attenuates beta-amyloid-induced neurotoxicity: reduction in oxidative damage through the Erk/P38 mitogen-activated protein kinase pathways. Antioxid Redox Signal 2013; 18: 158-169.

20. Maccarinelli F, Gammella E, Asperti M, Regoni M, Biasiotto G, Turco E et al. Mice lacking mitochondrial ferritin are more sensitive to doxorubicin-mediated cardiotoxicity. J Mol Med 2014; 92: 859-869.

21. Cacicedo JM, Gauthier MS, Lebrasseur NK, Jasuja R, Ruderman NB, Ido Y. Acute exercise activates AMPK and eNOS in the mouse aorta. Am J Physiol Heart Circ Physiol 2011; 301: $\mathrm{H} 1255-\mathrm{H} 1265$

22. Arosio $\mathrm{P}$, Levi S. Cytosolic and mitochondrial ferritins in the regulation of cellular iron homeostasis and oxidative damage. Biochim Biophys Acta 2010; 1800: 783-792.

23. Golstein P. Controlling cell death. Science 1997; 275: 1081-1082.

24. Zheng TS, Hunot S, Kuida K, Momoi T, Srinivasan A, Nicholson DW et al. Deficiency in caspase-9 or caspase-3 induces compensatory caspase activation. Nat Med 2000; 6: 1241-1247.

25. Wang X, Martindale JL, Liu Y, Holbrook NJ. The cellular response to oxidative stress: influences of mitogen-activated protein kinase signalling pathways on cell survival. Biochem J 1998; 333: 291-300

26. Orrenius S, Zhivotovsky B, Nicotera P. Regulation of cell death: the calcium-apoptosis link. Nat Rev Mol Cell Biol 2003; 4: 552-565.

27. Arosio P, Levi S. Ferritin, iron homeostasis, and oxidative damage. Free Radic Biol Med 2002; 33: 457-463.

28. Schieber M, Chandel NS. ROS function in redox signaling and oxidative stress. Curr Biol 2014; 24: R453-R462.

29. Brookes PS, Levonen AL, Shiva S, Sarti P, Darley-Usmar VM. Mitochondria: regulators of signal transduction by reactive oxygen and nitrogen species. Free Radic Biol Med 2002; 33: 755-764.

30. Campanella A, Rovelli E, Santambrogio P, Cozzi A, Taroni F, Levi S. Mitochondrial ferritin limits oxidative damage regulating mitochondrial iron availability: hypothesis for a protective role in Friedreich ataxia. Hum Mol Genet 2009; 18: 1-11.

31. Muthusamy VR, Kannan S, Sadhaasivam K, Gounder SS, Davidson CJ, Boeheme C et al. Acute exercise stress activates Nrf2/ARE signaling and promotes antioxidant mechanisms in the myocardium. Free Radic Biol Med 2012; 52: 366-376.

32. Kruman A II, Nath, Mattson MP. HIV-1 protein Tat induces apoptosis of hippocampal neurons by a mechanism involving caspase activation, calcium overload, and oxidative stress. Exp Neurol 1998; 154: 276-288.

33. Bush Al, Tanzi RE. Therapeutics for Alzheimer's disease based on the metal hypothesis. Neurotherapeutics 2008; 5: 421-432.

34. Bartnikas TB, Campagna DR, Antiochos B, Mulhern H, Pondarré C, Fleming MD. Characterization of mitochondrial ferritin-deficient mice. Am J Hematol 2010; 85: 958-960.

35. You LH, Li F, Wang L, Zhao SE, Wang SM, Zhang LL et al. Brain iron accumulation exacerbates the pathogenesis of MPTP-induced Parkinson's disease. Neuroscience 2015; 284: 234-246.

36. Hoene M, Lehmann R, Hennige AM, Pohl AK, Häring HU, Schleicher ED et al. Acute regulation of metabolic genes and insulin receptor substrates in the liver of mice by one single bout of treadmill exercise. J Physiol 2009; 587: 241-252.

37. Safdar A, Abadi A, Akhtar M, Hettinga BP, Tarnopolsky MA. miRNA in the regulation of skeletal muscle adaptation to acute endurance exercise in C57BI/6J male mice. PLoS One 2009; 4: e5610.

38. Kim TI, Lee YK, Park SG, Choi IS, Ban JO, Park HK et al. I-Theanine, an amino acid in green tea, attenuates beta-amyloid-induced cognitive dysfunction and neurotoxicity: reduction in oxidative damage and inactivation of ERK/p38 kinase and NF-kappaB pathways. Free Radic Biol Med 2009; 47: 1601-1610.

39. Huang W, Xie WB, Qiao D, Qiu P, Huang E, Li B et al. Caspase-11 plays an essential role in methamphetamine-induced dopaminergic neuron apoptosis. Toxicol Sci 2015; 145: 68-79. 